\title{
LATTICE EMBEDDINGS OF PRIME POWER GROUPS
}

D. W. BARNES

(received 28 July 1960)

\section{Introduction}

Let $G$ be a group of order $p^{n}$ where $p$ is some prime. Denote by $n_{r}(G)$ the number of subgroups of $G$ of order $p^{r}$. If $H$ is the elementary abelian group of order $p^{n}$, then

$$
n_{r}(G) \leqq n_{r}(H){ }^{*}
$$

This means that it is possible to construct a one-to-one mapping $f$ of the set of all subgroups of $G$ into the set of all subgroups of $H$ such that, for all subgroups $A \subseteq G$,

$$
|f|=|A| \text {. }
$$

This raises the problem of whether $f$ can be constructed so as to preserve the partial ordering of the subgroups of $G$, that is, such that $f A \subseteq f B$ whenever $A \subseteq B$.

An embedding of the lattice $L$ in a lattice $M$ is defined to be a one-to-one mapping $f$ of $L$ into $M$ such that $f X$ covers $f Y$ for all $X, Y \in L$ such that $X$ covers $Y$. If $G$ is a group, we denote the lattice of all subgroups of $G$ by $L(G)$. If $G$ and $H$ are groups of the same prime power order, we define a lattice embedding (which we abbreviate to $L$-embedding) of $G$ in $H$ to be an embedding of $L(G)$ in $L(H)$.

We say that the $L$-embeddings $f_{1}$ and $f_{2}$ of a prime power group $G$ in a group $H$ of the same order are similar if there exist automorphisms $\alpha, \beta$ of $G, H$ respectively such that

$$
f_{1}=\beta f_{2} \alpha,
$$

where we do not distinguish in notation between an automorphism of a group and the lattice automorphism it induces. By $\beta f_{2} \propto$ we mean the mapping formed by first applying the mapping $\alpha$, then $f_{2}$ and then $\beta$. We say $f_{1}$ and $f_{2}$ are equivalent if for some $\beta$ it is possible to take $\alpha=1$, that is, if there exists $\beta$ such that

$$
f_{1}=\beta f_{2} \text {. }
$$

- G. E. Wall [5]. 
This paper is an investigation of the groups $G$ and $H$ for which there exists an $L$-embedding of $G$ in $H$. We find necessary and sufficient conditions for an abelian $p$-group to have an $L$-embedding in the elementary abelian group of the same order.

Notation. Where possible, capital letters will be used for groups and lattices, while small letters will be used for elements of groups and for arbitrary integers. The group generated by $x_{1}, \cdots, x_{r}$ will be denoted by $\left\{x_{1}, \cdots, x_{r}\right\}$. We shall call a group $G$, a group of $r$ generators if there exists a set of $r$ elements of $G$ which generates $G$, and no set of fewer than $r$ elements of $G$ generates $G$. The intersection of all the maximal subgroups of a group $G$ will be denoted by $\Phi(G)$. If the group (or lattice) $A$ is isomorphic to the group (or lattice) $B$, we write $A \cong B$. We shall use the signs $\cup, \cap$ for the lattice operations of union and intersection. By $A \supset B$, we mean that $A$ contains $B$ and $A \neq B$. The image of $x$ under a mapping $f$ will be denoted by $f x$. Brackets will only be used when their omission would make the notation ambiguous.

The elementary abelian group of order $p^{n}$ is the additive group of a vector space of dimension $n$ over the field $G F(p)$ of $p$ elements. Its lattice is isomorphic to the lattice of subspaces of a projective geometry of dimension $n-1$ over $G F(p)$. We shall often use matrix and vector notations when working with this group. In this paper, only finite groups are considered. Whenever the word "group" is used, it is to be taken to mean "finite group".

$G$ and $H$ are always groups of the same prime power order.

THEOREM 1.1. Let $f$ be an embedding of a lower semi-modular lattice $L$ of finite dimension in a lattice $M$. Then for all $X, Y \in L$,

$$
f(X \cap Y)=(f X) \cap(f Y) .
$$

Proof. (a) Suppose $X \supseteqq Y$. Consider the image of a connected chain from $X$ to $Y$. This is a connected chain from $f X$ to $f Y$, therefore $f X \supseteqq f Y$ and the result holds.

(b) Suppose $X \cup Y$ covers $Y$. We prove the result for this case by induction over $d(X)+d(Y)$, where $d(X)$ is the dimension of $X$ in $L$, assuming $X \cup Y \neq X$.

There exists $Z \in L$ such that $X \cup Y$ covers $Z$ and $Z \supseteq X$. By the lower semi-modularity of $L, Y$ and $Z$ cover $Y \cap Z$. Hence

$$
f(Y \cap Z)=(f Y) \cap(f Z) \text { and } X \cup(Y \cap Z)=Z \text {. }
$$

Hence $f(X \cap Y)=f(X \cap(Y \cap Z))$

$$
\begin{aligned}
& =(f X) \cap f(Y \cap Z) \text { by induction hypothesis } \\
& =(f X) \cap(f Y) \cap(f Z) \\
& =(f X) \cap(f Y) .
\end{aligned}
$$

(c) We prove the theorem by induction over $d(X)+d(Y)$. By (a) and (b), we may assume $X \cup Y \neq X$ or $Y$ and $X \cup Y$ does not cover $Y$. 
There exists $Z \in L$ such that $X \cup Y$ covers $Z$ and $Z \supseteqq Y$. Then $X \cap Z \neq X$.

$$
\begin{aligned}
f(X \cap Y) & =f((X \cap Z) \cap Y) & & \\
& =f(X \cap Z) \cap(f Y) & & \text { by induction hypothesis } \\
& =(f X) \cap(f Z) \cap(f Y) & & \text { by (b) since } X \cup Z=X \cup Y \text { covers } Z \\
& =(f X) \cap(f Y) & & \text { since by (a) }, f Z \supseteqq f Y .
\end{aligned}
$$

CoROLLARY 1.2. If $f$ is an L-embedding of a p-group $G$ in a p-group $H$, then

for all $X, Y \subseteq G$.

$$
f(X \cap Y)=(f X) \cap(f Y)
$$

Proof. $L(G)$ is lower semi-modular since $G$ is a p-group.

COROLLARY 1.3. If $f$ is an L-embedding of $G$ in $H$ and $L(G)$ is modular, then $f(L(G))$ is a sublattice of $L(H)$.

Proof. Apply Theorem 1.1 to $L(G)$ and to its dual.

\section{Projectivities}

Suppose $A_{1}, B_{1}, A_{2}, B_{2}$ are subgroups of a group $G$, and that $B_{1}$ is a normal subgroup of $A_{1}, A_{1}=A_{2} \cup B_{1}$ and $B_{2}=B_{1} \cap A_{2}$. Then the correspondence between the cosets of $B_{1}$ in $A_{1}$ and their intersections with $A_{2}$ is an isomorphism $A_{1} / B_{1} \stackrel{\varphi_{1}}{\rightarrow} A_{2} / B_{2}$. The correspondence $\varphi_{2}$ between the cosets of $B_{2}$ in $A_{2}$ and their products with $B_{1}$ is also an isomorphism $A_{2} / B_{2} \stackrel{\varphi_{2}}{\rightarrow} A_{1} / B_{1}$, and $\varphi_{2}=\varphi_{1}^{-1}$. Such isomorphisms $\varphi_{1}, \varphi_{2}$ are called projectivities. The projectivities $\varphi_{1}, \varphi_{2}$ are called prime if $A_{1}$ covers $A_{2}$. The interval $A_{1} / B_{1}$ is called prime if $A_{1}$ covers $B_{1}$.

We call

$$
c: A_{1} / B_{1} \stackrel{\varphi_{1}}{\rightarrow} A_{2} / B_{2} \stackrel{\varphi_{2}}{\rightarrow} \cdots \stackrel{\varphi_{n-1}}{\rightarrow} A_{n} / B_{n}
$$

a chain of projectivities from $A_{1} / B_{1}$ to $A_{n} / B_{n}$ if all the mappings $\varphi_{i}$ are projectivities. The chain is called closed if $A_{1}=A_{n}$ and $B_{1}=B_{n}$. Two intervals $A / B, A^{\prime} \mid B^{\prime}$ are said to be projective if there exists a chain of projectivities from $A / B$ to $A^{\prime} / B^{\prime}$. If

$$
c: A / B \stackrel{\varphi_{0}}{\rightarrow} A_{1} / B_{1} \stackrel{\varphi_{1}}{\rightarrow} \cdots \stackrel{\varphi_{n-1}}{\rightarrow} A_{n} / B_{n} \stackrel{\varphi_{n}}{\rightarrow} A / B
$$

is a closed chain of projectivities, then $\varphi_{n} \varphi_{n-1} \cdots \varphi_{1} \varphi_{0}$ defines an automorphism of $A / B$. We call this automorphism, the automorphism induced by $c$, and denote it by $\alpha(c)$. If

$$
c^{\prime}: A / B \rightarrow A_{1}^{\prime} / B_{1}^{\prime} \rightarrow \cdots \rightarrow A_{r}^{\prime} / B_{r}^{\prime} \rightarrow A / B
$$

is another closed chain of projectivities from $A / B$ to $A / B$, we define $c c^{\prime}$ to be the closed chain of projectivities

$c c^{\prime}: A \mid B \rightarrow A_{1} / B_{1} \rightarrow \cdots \rightarrow A_{n} / B_{n} \rightarrow A / B \rightarrow A_{1}^{\prime} / B_{1}^{\prime} \rightarrow \cdots \rightarrow A_{r}^{\prime} / B_{r}^{\prime} \rightarrow A / B$, and 
Clearly

$$
c^{-1}: A / B \rightarrow A_{n} / B_{n} \rightarrow \cdots \rightarrow A_{1} / B_{1} \rightarrow A / B .
$$

$$
\alpha\left(c c^{\prime}\right)=\alpha\left(c^{\prime}\right) \alpha(c), \quad \alpha\left(c^{-1}\right)=\alpha(c)^{-1} .
$$

The automorphisms induced in $A / B$ by closed chains of projectivities in $G$ form a group $\mathfrak{A}(G, A / B)$. If $A / B$ and $A^{\prime} / B^{\prime}$ are projective, then

$$
\mathfrak{A}(G, A / B) \cong \mathfrak{A}\left(G, A^{\prime} / B^{\prime}\right) .
$$

THEOREM 2.1. If $G$ is a p-group with more than one subgroup of order $p$, then all prime intervals of $G$ are projective.*

Proof. The theorem is trivial if $G$ is elementary abelian. We suppose that $G$ is of order greater than $p^{2}$ and that the theorem holds for all groups of smaller order than $G$. Since $G$ has at least two subgroups of order $p$, and one of these may be taken to be in the centre, $G$ has an elementary abelian subgroup $X$ of order $p^{2}$. Let $M$ be any maximal subgroup of $G$ which contains $X$. Then all prime intervals of $M$ are projective. Since $G / \Phi$ is elementary abelian of order at least $p^{2}$, and $M \supset \Phi$, all prime intervals of $G / \Phi$ are projective to all prime intervals of $M$. It is sufficient to prove that any prime interval, $A / B$ of $G$ is projective to an interval of $G / M$ or of $M$. Take any composition series from $G$ to 1 through $M$. By Zassenhaus's Lemma, $A / B$ is projective to some factor of this composition series.

\section{The First Canonicity Condition}

Let $f$ be an $L$-embedding of a $p$-group $G$ in a group $H$ of the same order. Let

$$
c: A_{1} / B_{1} \rightarrow A_{2} / B_{2} \rightarrow \cdots \rightarrow A_{1} / B_{1}
$$

be a closed chain of projectivities in $G$ with $A_{i} / B_{i}$ cyclic of order $p$. Then $f B_{i}$ is normal in $f A_{i}, f A_{i} / f B_{i}$ is cyclic of order $p$, and

$$
\bar{c}: f A_{1} / f B_{1} \rightarrow f A_{2} / f B_{2} \rightarrow \cdots \rightarrow f A_{1} / f B_{1}
$$

is a closed chain of projectivities in $H$. If $x, \bar{x}$ are any elements of $A_{1} / B_{1}$, $f A_{1} / f B_{1}$ respectively, then

$$
\alpha(c) x=x^{r(o)}, \quad \bar{\alpha}(\bar{c}) \bar{x}=\bar{x}^{\bar{r}(\bar{e})}
$$

where $r, \bar{r}$ are integers $\bmod p,(r, p)=(\bar{r}, p)=1$ and $r, \bar{r}$ are independent of $x, \bar{x}$.

We say that the $L$-embedding $f$ is 1 -canonical if, for all closed chains $c$ of projectivities on cycles of order $p$ in $G$,

$$
r(c) \equiv \bar{r}(\bar{c}) \quad(\bmod p) .
$$

We shall often find it more convenient to consider individual projectivities than closed chains. So we construct a system which will enable us to

* I am indebted to Dr. O. Tamaschke for a simplification of my original proof. 
deduce from its satisfying a condition for each projectivity, that the $L$ embedding under consideration is 1-canonical. For each prime interval $A_{i} / B_{i}$ of $G$, we choose a generator $a_{i}$ of $A_{i} / B_{i}$ and a generator $\bar{a}_{i}$ of $f A_{i} / f B_{i}$. We call the set $\left(a_{i}, \bar{a}_{i}\right)$ a basis of $f$. The projectivity $A_{i} / B_{i} \rightarrow A_{j} / B_{j}$ is called a regular projectivity of the basis $\left(a_{i}, \tilde{a}_{i}\right)$ if, in the isomorphisms $a_{i} \rightarrow a_{j}^{r}$, $\bar{a}_{i} \rightarrow \bar{a}_{j}^{s}$ defined by the projectivities $A_{i} / B_{i} \rightarrow A_{j} / B_{j}, f A_{i} / f B_{i} \rightarrow f A_{j} / f B_{j}$ respectively, $r \equiv s(\bmod p)$.

Clearly, if $A_{1} \supset A_{2} \supset A_{3}$ and any two of the projectivities $A_{1} / B_{1} \rightarrow A_{2} / B_{2}$, $A_{1} / B_{1} \rightarrow A_{3} / B_{3}$ and $A_{2} / B_{2} \rightarrow A_{3} / B_{3}$ are regular, then so is the third. We shall refer to this as "the three intervals rule".

The basis $\left(a_{i}, \bar{a}_{i}\right)$ of $f$ is called canonical if every projectivity is regular. To prove a basis canonical, by the three intervals rule, it is clearly sufficient to prove that all prime projectivities are regular. If the $L$-embedding $f$ has a canonical basis, then clearly $f$ is 1-canonical. Conversely, if $f$ is a 1-canonical embedding, then there exists a canonical basis of $f$, although in general, not all bases of $f$ will be canonical.

The significance of the first canonicity condition may be seen from the following theorem.

THEOREM 3.1. An L-embedding $f$ of a p-group $G$ in a group $H$ of the same order can be extended to an L-embedding $F$ of $G \times C$ in $H \times \bar{C}(F X=f X$ for $X \subseteq G$ ) where $C, C$ are cyclic of order $p$, if and only if $f$ is 1-canonical.

Proof. (1) Suppose $F$ is an extension. Let

$$
c: A_{1} / B_{1} \rightarrow A_{2} / B_{2} \rightarrow \cdots \rightarrow A_{1} / B_{1}
$$

be any closed chain of projectivities in $G$ on prime intervals. We have to prove that $r(c) \equiv \bar{r}(\bar{c})(\bmod p)$.

Since if $c^{\prime}$ is the chain $A_{2} / B_{2} \rightarrow A_{3} / B_{3} \rightarrow \cdots \rightarrow A_{1} / B_{1} \rightarrow A_{2} / B_{2}$, $r(c) \equiv r\left(c^{\prime}\right)$ and $\bar{r}(\bar{c}) \equiv \bar{r}\left(\bar{c}^{\prime}\right)$, we need only consider the case $A_{2} \supset A_{1}$. Further, we may assume that $A_{2}$ covers $A_{1}$, for if not, we can insert $A_{2}^{\prime} / B_{2}^{\prime}$ such that $A_{2} \supset A_{2}^{\prime}, A_{2}^{\prime}$ covers $A_{1}$ and

$$
c^{\prime \prime}: A_{1} / B_{1} \rightarrow A_{2}^{\prime} / B_{2}^{\prime} \rightarrow A_{2} / B_{2} \rightarrow \cdots \rightarrow A_{1} / B_{1}
$$

is a chain of projectivities in $G$ with $r(c) \equiv r\left(c^{\prime \prime}\right)$ and $\bar{r}(\bar{c}) \equiv \bar{r}\left(\bar{c}^{\prime \prime}\right)$.

Take generators $a, b, c$ of $A_{1} / B_{1}, B_{2} / B_{1}$ and $B_{1} \times C / B_{1}$ respectively, and choose generators $\bar{a}, \bar{b}, \bar{c}$ of $F A_{1} / F B_{1}, F B_{2} / F B_{1}, F\left(B_{1} \times C\right) / F B_{1}$ such that in the restriction of $F$ to $A_{2} \times C / B_{1}$,

$$
F\{a b c\}=\{\bar{a} \bar{b} \bar{c}\} .
$$

Since $L\left(\left(A_{2} \times C\right) / B_{1}\right)$ and $L\left(F\left(A_{2} \times C\right) / F B_{1}\right)$ are plane projective geometries over $G F(p)$, mappings of $L\left(\left(A_{2} \times C\right) / B_{1}\right)$ onto $L\left(F\left(A_{2} \times C\right) / F B_{1}\right)$ are linear and are determined by the images of the vertices of a triangle of reference and the image of the unit point. Therefore 


$$
F\left\{a^{x} b^{y} c^{z}\right\}=\left\{\bar{a}^{x} b^{y} \bar{c}^{z}\right\}
$$

Consider the closed chain of projectivities

$$
\gamma: A_{1} \times C / B_{1} \rightarrow A_{2} \times C / B_{2} \rightarrow \cdots \rightarrow A_{1} \times C / B_{1} .
$$

This induces in $A_{1} \times C / B_{1}$ the automorphism $\alpha(\gamma) a^{x} c^{z}=a^{x r(e)} c^{x}$. $\bar{\gamma}$ induces in $F\left(A_{1} \times C\right) / F B_{1}$ the automorphism $\bar{\alpha}(\bar{\gamma}) \bar{a}^{x} \bar{c}^{z}=\bar{a}^{x \bar{x}(\bar{c})} \bar{c}^{z}$. But $F \alpha(\gamma)\left\{a^{x} c^{z}\right\}=\bar{\alpha}(\bar{\gamma}) F\left\{a^{x} c^{z}\right\}$. Therefore $\left\{\tilde{a}^{x r(c)} \bar{c}^{z}\right\}=\left\{\bar{a}^{x \bar{r}(\bar{c})} \bar{c}^{z}\right\}$. Therefore $r(c) \equiv \bar{r}(\bar{c})(\bmod p)$.

(2) Suppose $f$ is 1 -canonical. Take a canonical basis of $f$ and generators $c, \bar{c}$ of $C, \bar{C}$.

For all $A \subseteq G$, define $F A=f A, F(A \cup C)=F A \cup C$. Suppose $X$ is a subgroup of $G \times C$ for which we have not yet defined $F X$. Then $X \cup C$ covers $X$ covers $X \cap G, X \cap G$ is normal in $X \cup C$ and $(X \cup C) /(X \cap G)$ is elementary abelian of order $p^{2}$. $(X \cup C) /(X \cap G)$ is generated by the element $a \in\left(a_{i}, \bar{a}_{i}\right)$ corresponding to $((X \cup C) \cap G) /(X \cap G)$ and the coset $c^{\prime}=c(X \cap G)$. Similarly $F(X \cup C) / F(X \cap G)$ is generated by the element $\bar{a} \epsilon\left(a_{i}, \bar{a}_{i}\right)$ corresponding to $F((X \cup C) \cap G) / F(X \cap G)$ and the coset $\bar{c}^{\prime}=\bar{c} F(X \cap G)$. If $x$ is a generator of $X /(X \cap G)$, then $x=a^{r} c^{\prime s}$. Let $\bar{x}=\bar{a}^{r} \bar{c}^{\prime s}$ and define $F X$ by $F X \mid F(X \cap G)=\{\bar{x}\}$.

To prove that $F$ so defined is an $L$-embedding of $G \times C$ in $H \times \bar{C}$, we have only to prove that, if $Y$ is a subgroup of $G \times C, G \neq Y \neq C$ and $X$ covers $Y$, then $F X$ covers $F Y$.

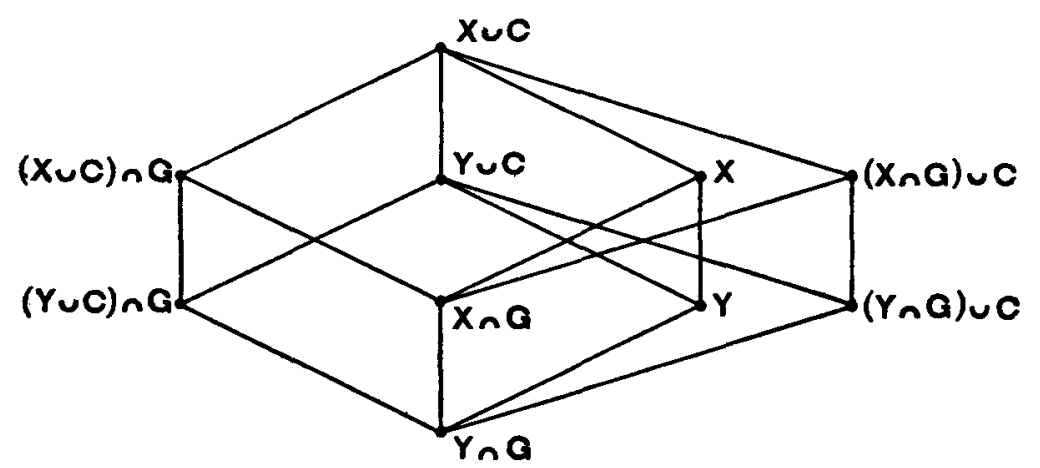

Since $(X \cup C) \cap G, Y \cup C, X$ are maximal subgroups of $X \cup C$, $((X \cup C) \cap G) \cap(Y \cup C) \cap X=Y \cap G$ is normal in $X \cup C$ and $(X \cup C) /(Y \cap G)$ is elementary abelian of order $p^{3}$. Take generators $u, v, w$ of $((Y \cup C) \cap G) /(Y \cap G), \quad(X \cap G) /(Y \cap G), \quad((Y \cap G) \cup C) /(Y \cap G)$ respectively, $u, v \in\left(a_{i}, \bar{a}_{i}\right), \quad w=c(Y \cap G)$ and generators $\bar{u}, \bar{v}, \bar{v}$ of $F((Y \cup C) \cap G) / F(Y \cap G), F(X \cap G) / F(Y \cap G), F((Y \cap G) \cup C) / F(Y \cap G)$ respectively, with $\bar{u}, \bar{v} \in\left(a_{i}, \bar{a}_{i}\right)$ and $\bar{w}=\bar{c} F(Y \cap G)$.

In the isomorphism $\varphi$ of $(X \cup C) /(Y \cap G)$ onto $F(X \cup C) / F(Y \cap G)$ defined by $\varphi u^{i} v^{j} w^{k}=\bar{u}^{i} \bar{v}^{j} \bar{w}^{k}$, 
$\varphi X=\varphi\left\{a^{r} c^{\prime s}, v\right\}$

$=\varphi\left\{u^{n r} w^{s}, v\right\}$ where $n$ is given by $a \rightarrow u^{n}$ in the projectivity

$=\left\{\bar{u}^{n r} \bar{w}^{s}, \bar{v}\right\}$

$((X \cup C) \cap G) /(X \cap G) \rightarrow((Y \cup C) \cap G) /(Y \cap G)$

$=\left\{\bar{a}^{r} \bar{c}^{\prime \prime}, \bar{v}\right\}$

since $((X \cup C) \cap G) /(X \cap G) \rightarrow((Y \cup C) \cap G) /(Y \cap G)$

$=F X$. is a regular projectivity of $\left(a_{i}, \bar{a}_{i}\right)$

Trivially $\varphi Y=F Y$ and therefore $F X$ covers $F Y$.

We call the extension constructed in the above manner, the standard extension of $f$. We now show that, except in some trivial exceptional cases, the standard extension is the only extension.

THEOREM 3.2. Let $G$ be a p-group with more than one subgroup of order $p$, and let $f$ be an L-embedding of $G$ in a group $H$ of the same order. Let $C, \bar{C}$ be cyclic groups of order p. If $F_{1}$ and $F_{2}$ are extensions of $f$ to $L$-embeddings of $G \times C$ in $H \times \bar{C}$ such that $F_{1}(C)=F_{2}(C)=\bar{C}$, then $F_{1}=\alpha F_{2}$ where $\alpha$ is an automorphism of $H \times C$ of the form $\alpha h \bar{c}=h \bar{c}^{r}$ for all $h \in H, \bar{c} \in C$ where $r$ is an integer independent of $h, \bar{c}$ with $(r, p)=1$.

Proof. (1) If for some $X, G \neq X \neq C, F_{1} X=F_{2} X$, then $F_{1}$ and $F_{2}$ coincide on $(X \cup C) /(X \cap G)$. This is true because $((X \cup C) \cap G) /(X \cap G)$ is projective in $G$ to some other interval of $G$, and therefore there exist $X^{\prime}, X^{\prime \prime}$ such that $X^{\prime} \supseteqq X \supseteqq X^{\prime \prime}$ and $\left(X^{\prime} \cup C\right) /\left(X^{\prime \prime} \cap G\right)$ is elementary abelian of order $p^{3}$. A mapping of $L\left(\left(X^{\prime} \cup C\right) /\left(X^{\prime \prime} \cap G\right)\right)$ onto

$$
L\left(F_{1}\left(X^{\prime} \cup C\right) / F_{1}\left(X^{\prime \prime} \cap G\right)\right)=L\left(F_{2}\left(X^{\prime} \cup C\right) / F_{2}\left(X^{\prime \prime} \cap G\right)\right)
$$

must be linear and hence the mapping of $L((X \cup C) /(X \cap G))$ is determined by the images of the three points $X,(X \cup C) \cap G,(X \cap G) \cup C$.

(2) If for some $X \subseteq G \times C, G \notin X \neq C, F_{1}$ and $F_{2}$ coincide on $(X \cup C) /(X \cap G)$, then $F_{1}=F_{2}$. This is because, by Theorem 2.1, for any $Y \subseteq G \times C, G \notin Y \nsubseteq C$, there exists a chain of projectivities

$$
(X \cup C) /(X \cap G) \rightarrow\left(X_{1} \cup C\right) /\left(X_{1} \cap G\right) \rightarrow \cdots \rightarrow(Y \cup C) /(Y \cap G)
$$

which determines uniquely the mapping of $L((Y \cup C) /(Y \cap G))$ from the mapping of $L((X \cup C) /(X \cap G))$.

(3) Let $g \in G$ be an element of order $p$ and let $\bar{g}$ be a generator of $f\{g\}$. Let $c, \bar{c}$ be generators of $C, C$ respectively. Then for some $m, n, F_{1}\{g c\}=\left\{\bar{g} \bar{c}^{m}\right\}$ and $F_{2}\{g c\}=\left\{\bar{g} \bar{c}^{n}\right\}$. If $\alpha$ is the automorphism of $H \times C: \alpha h \bar{c}^{k}=h \bar{c}^{r k}$ where $h$ is any element of $H$ and $r n \equiv m(\bmod p)$, then $F_{1}\{g c\}=\alpha F_{2}\{g c\}$ and, by (1) and (2), $F_{1}=\alpha F_{2}$.

When $G$ is a group with only one subgroup of order $p$, this subgroup is not projective to any other prime interval of $G$, and consequently the mapping of the lattice of its direct product with $C$ need not be linear and can be con- 
structed independently of the construction of the mapping of the rest of the lattice of $G \times C$. This situation arises only when $G$ is cyclic of odd prime order as an $L$-embedding of an elementary abelian group of order 4 is determined by the images of two subgroups of order 2 .

THEOREM 3.3. The standard extension $F$ of a 1-canonical L-embedding $f$ of a p-group $G$ in a group $H$ of the same order to an L-embedding of $G \times C$ in $H \times \bar{C}$, where $C, C$ are cyclic of order $p$, is 1-canonical.

Proof. We extend the canonical basis $\left(a_{i}, \bar{a}_{i}\right)$ of $f$ used in the construction of $F$ to a canonical basis of $F$. Let $c, \bar{c}$ be the generators of $C, C$ used in the construction of $F$. For every $A \subseteq G$, take as the basis elements for $(A \cup C) / A$, the cosets $c A, \bar{c} F A$. If $A$ covers $B$ and $a, \bar{a}$ are the basis elements for $A / B$, take $a(B \cup C), \vec{a} F(B \cup C)$ as the basis elements for $(A \cup C) /(B \cup C)$. For every $X \subset G \times C, G \notin X \neq C$, take as basis elements for $X /(X \cap G), x$ and $\bar{x}$ as defined in the construction of $F X$. For $(X \cup C) / X$, take as basis elements $c X, \bar{c} F X$. If $X$ covers $Y, Y \notin G$, then take $v Y, \bar{v} F Y$ as the basis elements for $X / Y$, where $v, \bar{v}$ are elements of $\left(a_{i}, \bar{a}_{i}\right)$ for $(X \cap G) /(Y \cap G)$.

Clearly, all projectivities not involving a subgroup $X, G \notin X \notin C$ are regular. Since for every such $X$, there exists an isomorphism of $(X \cup C) /(X \cap G)$ onto $F(X \cup C) / F(X \cap G)$ which maps basis elements onto corresponding basis elements, all projectivities within an interval $(X \cup C) /(X \cap G)$ are regular. If $X$ covers $Y, Y \notin G$, then there exists an isomorphism (given in the proof of the existence of $F)$ of $(X \cup C) /(Y \cap G)$ onto $F(X \cup C) / F(Y \cap G)$ which maps basis elements onto corresponding basis elements. Hence all projectivities within $(X \cup C) /(Y \cap G)$ are regular. The only remaining prime projectivities are of the form $X / Y \rightarrow X^{\prime} / Y^{\prime}$, where $X^{\prime} \notin C, Y^{\prime} \ddagger G$. Suppose $X$ covers $X^{\prime}$. Then $X \cup C \supset X^{\prime} \cup C \supset X^{\prime}$ and $(X \cup C) /(Y \cup C) \rightarrow\left(X^{\prime} \cup C\right) /\left(Y^{\prime} \cup C\right)$ and $\left(X^{\prime} \cup C\right) /\left(Y^{\prime} \cup C\right) \rightarrow X^{\prime} / Y^{\prime}$ are regular. Therefore, by the three intervals rule, $(X \cup C) /(Y \cup C) \rightarrow X^{\prime} / Y^{\prime}$ is regular. But $X \cup C \supset X \supset X^{\prime}$ and $(X \cup C) /(Y \cup C) \rightarrow X / Y$ is regular. By the three intervals rule, $X / Y \rightarrow X^{\prime} / Y^{\prime}$ is regular. Hence all prime projectivities are regular and $F$ is therefore 1-canonical.

\section{Two Generator Groups}

Lemma 4.1. Let $\left(a_{i}, \bar{a}_{i}\right)$ be a basis of an L-embedding $f$ of a p-group $G$ in a group $H$ of the same order. Suppose that, for $G / \Phi$ and for each maximal subgroup of $G,\left(a_{i}, \bar{a}_{i}\right)$ gives a canonical basis for the corresponding restriction of $f$. Then $f$ is 1-canonical and $\left(a_{i}, \bar{a}_{i}\right)$ is a canonical basis of $f$.

Proof. Consider a prime projectivity $A_{1} / B_{1} \rightarrow A_{2} / B_{2}$ of a prime interval $A_{1} / B_{1}$ of $G$. We may assume $A_{1}$ covers $A_{2}$. Either (1) there exists a maximal subgroup $M$ of $G$ with $M \supseteqq A_{1}$ and the projectivity is regular since the 
basis is canonical for the restriction of $f$ to $M$, or (2) $A_{1}=G$ and $A_{2}, B_{1}$ are maximal subgroups of $G$. Then $B_{2}=A_{2} \cap B_{1} \supseteqq \Phi$ and the projectivity is regular since the basis is canonical for the restriction of $f$ to $G / \Phi$. Therefore all prime projectivities are regular and $\left(a_{i}, \bar{a}_{i}\right)$ is a canonical basis of $f$.

THEOREM 4.2. Let $G$ be a p-group all of whose subgroups are groups of at most two generators, and let $H$ be an elementary abelian group of the same order as $G$. Let $K$ and $\bar{K}$ be subgroups of the same order of $G$ and $H$ respectively, and let $f$ be a 1-canonical L-embedding of $K$ in $\bar{K}$. Then there exists a 1-canonical extension $F$ of $f$ to an L-embedding of $G$ in $H$.

Proof. We may assume that $K$ is a maximal subgroup of $G$. The theorem is trivial if $G$ is of order $p^{2}$. We assume that, for groups of smaller order than $G$, an $L$-embedding of a maximal subgroup with a given canonical basis can be extended to an $L$-embedding of the group such that the given basis can be extended to a canonical basis of the extended $L$-embedding with prescribed basis elements for the factor group of the maximal subgroup.

Since $G / \Phi(G)$ is of order $p^{2}$, we can define $F$ on $G / \Phi(G)$ and the elements of a canonical basis of $F$ on $G / \Phi(G)$ having as the basis elements for $K / \Phi(G)$ the elements of the given basis, and any arbitrarily assigned basis elements for $G / K$. Since $\Phi(G)$ is a maximal subgroup of each of the maximal subgroups of $G$, and a subgroup of $G$ not contained in $\Phi(G)$ is contained in only one maximal subgroup of $G$, we may apply the induction hypothesis to the maximal subgroups of $G$, defining $F$ and the' basis elements independently for each maximal subgroup. By Lemma 4.1, the $L$-embedding so constructed is 1 -canonical and the basis constructed is canonical.

COROLLARY 4.3. If $G$ is a p-group with at most one proper subgroup of more than two generators, then $G$ has a 1-canonical L-embedding in the elementary abelian group of the same order.

Proof. (1) Suppose $G$ is a group of three generators, all of whose proper subgroups are of at most two generators. Then a subgroup of $G$ not containing and not contained in $\Phi(G)$ is contained in exactly one subgroup of $G$ of index $p^{2}$. Hence a 1-canonical $L$-embedding of a maximal subgroup $M$ of $G$ can be extended to the elementary abelian group $G / \Phi(G)$ with elements of canonical bases of the embeddings of $M$ and $G / \Phi(G)$ coinciding for the common intervals, and the procedure of the proof of theorem 4.1 may be applied independently to the remaining subgroups of index $p^{2}$.

(2) Suppose $G$ has a proper subgroup $K$ of three generators. We may assume that this subgroup is a maximal subgroup. By (1) it has a 1-canonical $L$-embedding. As before, we can extend this to $G / \Phi(G)$ and apply the procedure used above to the subgroups which cover $\Phi(G)$, since every $A \subset G$, $A \nsubseteq K, A \nsubseteq \Phi(G)$ is contained in exactly one subgroup of $G$ which covers $\Phi(G)$. 
CoROLlaRY 4.4. If $G$ is an abelian p-group of two generators, then $G$ has a 1-canonical L-embedding in the elementary abelian group.

\section{The nth Canonicity Condition $(n>1)$}

Let $f$ be an $L$-embedding of a $p$-group $G$ in the elementary abelian group $\bar{G}$ of the same order. We say that $f$ is $n$-canonical $(n>1)$ if, for all closed chains $c$ of projectivities in $G$ on cyclic factors of order $p^{n}, \alpha(c)=1$ if and only if $\bar{\alpha}(\bar{c})=\overline{1}$ where $1, \overline{1}$ are the identity automorphisms of the corresponding factors.

Throughout this section, $G$ shall denote a $p$-group and $\bar{G}$ the elementary abelian group of the same order. $C$ shall denote a cyclic group of order $p^{n}$ and $\bar{C}_{1}, \cdots, \bar{C}_{n}$ cyclic groups of order $p$. If $A$ is a group, we denote by $A^{r}$ the group generated by the $r$ th powers of the elements of $A$. We consider the problem of extending an $L$-embedding $f$ of $G$ in $\bar{G}$ to an $L$-embedding of $G \times C$ in $\bar{G} \times \bar{C}_{1} \times \cdots \times \bar{C}_{n}$.

Lemma 5.1. If $G \times C \supset X, G \times C^{p} \notin X \notin C^{p^{n-1}}$, then $X \cap G$ is normal in $X \cup C$ and $(X \cup C) /(X \cap G)$ is abelian of type $(n, n)$.

The proof is obvious.

LEMMA 5.2. Suppose $F X$ is defined for all $X \subseteq G \times C^{p}$ and for all $X \supseteqq C^{p^{n-1}}$ such that $F$ defines an L-embedding of $G \times C^{p}$ in $\bar{G} \times \bar{C}_{1} \times \cdots \times \bar{C}_{n-1}$ and of $(G \times C) / C^{p^{n-1}}$ in $\left(\bar{G} \times \bar{C}_{1} \times \cdots \times C_{n}\right) / C_{1}$. Then $F$ can be extended to an L-embedding of $G \times C$ in $\bar{G} \times C_{1} \times \cdots \times C_{n}$ if and only if the following condition holds:

for subgroups $X$ such that $G \times C^{p} \notin X \neq C^{p^{n-1}}$ and for closed chains of projectivities

$$
\gamma:(X \cup C) /(X \cap G) \rightarrow\left(X_{1} \cup C\right) /\left(X_{1} \cap G\right) \rightarrow \cdots \rightarrow(X \cup C) /(X \cap G),
$$

$\gamma$ induces the identity lattice automorphism in $\left(X \cup C^{p^{n-1}}\right) /\left(X \cap\left(G \times C^{p}\right)\right)$ if and only if $\bar{\gamma}$ induces the identity lattice automorphism in $F\left(X \cup C^{p^{n-1}}\right) / F\left(X \cap\left(G \times C^{p}\right)\right)$.

Proof. Let $\lambda(\gamma), \bar{\lambda}(\bar{\gamma})$ be the lattice automorphisms induced in $\left(X \cup C^{p^{n-1}}\right) /\left(X \cap\left(G \times C^{p}\right)\right)$ and $F\left(X \cup C^{p^{n-1}}\right) / F\left(X \cap\left(G \times C^{p}\right)\right)$ by $\gamma$ and $\bar{\gamma}$ respectively. If $F X$ for any one subgroup $X, G \times C^{p} \neq X \neq C^{n^{n-1}}$, is chosen arbitrarily from the subgroups of $F\left(X \cup C^{p^{n-1}}\right) / F\left(X \cap\left(G \times C^{p}\right)\right)$ not already images under $F$, the definition of $F$ may be extended by projectivities. $F$ can be extended to an $L$-embedding of $G \times C$ if and only if this procedure does not lead to contradictory definitions of $F$ for any subgroup. Thus $F$ can be extended to an $L$-embedding of $G \times C$ if and only if the correspondence $\lambda(\gamma) \leftrightarrow \bar{\lambda}(\bar{\gamma})$ between the lattice automorphisms induced in $\left(X \cup C^{p^{n-1}}\right) /\left(X \cap\left(G \times C^{p}\right)\right)$ and $F\left(X \cup C^{p^{n-1}}\right) / F\left(X \cap\left(G \times C^{p}\right)\right)$ by closed chains of projectivities $\gamma, \bar{\gamma}$ of the above form is one-to-one. The condition 
that the correspondence be one-to-one is that $\lambda(\gamma)$ be the identity if and only if $\bar{\lambda}(\bar{\gamma})$ is the identity.

LEMMA 5.3. In the notation of Lemma 5.2, $\gamma$ induces the identity lattice automorphism in $\left(X \cup C^{p^{n-1}}\right) /\left(X \cap\left(G \times C^{p}\right)\right)$ if and only if $\gamma$ induces the identity automorphism in $((X \cup C) \cap G) /(X \cap G)$.

Proof. Let $((X \cup C) \cap G) /(X \cap G)=\{a\},((X \ldots G) \cup C) /(X \cap G)=\{c\}$ and $X /(X \cap G)=\{x\}$. Then $a$ and $c$ generate $(X \cup C) /(X \cap G)$ which is abelian of type $(n, n)$. The generator $x$ of $X /(X \cap G)$ may be chosen such that $x=a^{k} c, p \nmid k$. Let

$$
\gamma:(X \cup C) /(X \cap G) \rightarrow\left(X_{1} \cup C\right) /\left(X_{1} \cap G\right) \rightarrow \cdots \rightarrow(X \cup C) /(X \cap G)
$$

be any closed chain of projectivities on factors $\left(X_{i} \cup C\right) /\left(X_{i} \cap G\right)$ with $G \times C^{p} \neq X_{i} \notin C^{p^{n-1}}$ mapping $X \cup C^{p^{n-1}}$ and $X \cap\left(G \times C^{p}\right)$ onto themselves. Then $\alpha(\gamma) c=c$ and $\alpha(\gamma) a=a^{s}, p \nmid s . \alpha(\gamma)\left\{a^{k} c\right\}=\left\{a^{s k} c\right\}$. Therefore $\gamma$ maps $X$ onto itself if and only if $s \equiv 1\left(\bmod p^{n}\right)$, which proves the lemma.

Lemma 5.4. In the notation of Lemma 5.2, $\bar{\gamma}$ induces the identity lattice automorphism in $F\left(X \cup C^{p^{n-1}}\right) / F\left(X \cap\left(G \times C^{p}\right)\right)$ if and only if $\bar{\gamma}$ induces the identity automorphism in $F((X \cup C) \cap G) / F(X \cap G)$.

Proof. Choose a basis $e_{1}, \cdots, e_{2 n}$ of $F(X \cup C) / F(X \cap G)$ such that

and

$$
\begin{aligned}
& F\left((X \cap G) \cup C^{n^{n-r}}\right)=\left\{e_{1}, \cdots, e_{r}\right\} \\
& F\left(\left(X \cup C^{p^{n-r}}\right) \cap G\right)=\left\{e_{n+1}, \cdots, e_{n+r}\right\}
\end{aligned}
$$

$$
F\left(X^{p^{n-1}} \cup(X \cap G)\right)=\left\{e_{1}+e_{n+1}, \cdots, e_{r}+e_{n+r}\right\} .
$$

This may be done however $F X$ is defined. For any $\gamma, \bar{\alpha}(\bar{\gamma})$ is given by a matrix of the form

$$
M=\left[\frac{I \mid O}{O \mid N}\right]
$$

where the submatrices are $n \times n$, since $\vec{\alpha}(\bar{\gamma})$ is the identity on $F((X \cap G) \cup C) / F(X \cap G)$ and maps $F((X \cup C) \cap G) / F(X \cap G)$ onto itself.

Let $N_{r}$ be the submatrix of $N$ consisting of the elements in the first $r$ rows and columns of $N$. Clearly, if $N=I$, then $\bar{\gamma}$ induces the identity lattice automorphism in $F\left(X \cup C^{p^{n-1}}\right) / F\left(X \cap\left(G \times C^{D}\right)\right)$. Suppose $\bar{\gamma}$ induces the identity lattice automorphism in $F\left(X \cup C^{p^{n-1}}\right) / F\left(X \cap\left(G \times C^{p}\right)\right)$. We have to prove that $N=I$.

Since $M$ maps $\left\{e_{1}\right\},\left\{e_{n+1}\right\},\left\{e_{1}+e_{n+1}\right\}$ onto themselves, it follows that $N_{1}=I$. We use induction over $r$. Suppose $N_{r}=I$.

Since $\left\{e_{n+1}, \cdots, e_{n+r}\right\}$ maps into itself, 


$$
\begin{gathered}
N_{r+1}=\left[\begin{array}{c|c}
I & a_{1} \\
& \vdots \\
\hline 0 \cdots 0 & \frac{a_{r}}{a_{r+1}}
\end{array}\right] . \\
M\left(e_{r+1}+e_{n+r+1}\right)=e_{r+1}+a_{1} e_{n+1}+\cdots+a_{r+1} e_{n+r+1} \\
\equiv \lambda\left(e_{r+1}+e_{n+r+1}\right) \bmod \left\{e_{1}+e_{n+1}, \cdots, e_{r}+e_{n+r}\right\} .
\end{gathered}
$$

Therefore $\lambda=1, a_{1}=a_{2}=\cdots=a_{r}=0$ and $a_{r+1}=1$ which proves the lemma.

Lemma 5.5. Suppose $F X$ is defined for all $X \subseteq G \times C^{p}$ and for all $X \supseteqq C^{p^{n-1}}$ such that $F$ defines an L-embedding of $G \times C^{p}$ in $\bar{G} \times \bar{C}_{1} \times \cdots \times \bar{C}_{n-1}$ and of $(G \times C) / C^{p^{n-1}}$ in $\left(\bar{G} \times \bar{C}_{1} \times \cdots \times \bar{C}_{n}\right) / \bar{C}_{1}$. Let $f$ be the restriction of $F$ to $G$. Then $F$ can be extended to an L-embedding of $G \times C$ in $\bar{G} \times \bar{C}_{1} \times \cdots \times \bar{C}_{n}$ if and only if $f$ is $n$-canonical, $n \geqq 1$.

Proof. The case $n=1$ has been proved in Theorem 3.1. For $n>1$, the result follows from Lemmas 5.2, 5.3, and 5.4.

ThEOREM 5.6. An L-embedding $f$ of a p-group $G$ in the elementary abelian group $\bar{G}$ of the same order can be extended to an L-embedding $F$ of $G \times C$ in $\bar{G} \times \bar{C}_{1} \times \cdots \times \bar{C}_{n}$, where $C$ is cyclic of order $p^{n}$ and $\bar{C}_{i}$ are of order $p$, if and only if $f$ is 1-, 2-, $\cdots, n$-canonical.

Proof. If $F$ exists, then by Lemma 5.5, $f$ is $1-, 2-, \cdots, n$-canonical. Suppose $f$ is $1-, 2-, \cdots, n$-canonical. We have to prove that there exists an extension $F$.

Let $\varphi_{i}$ be the isomorphism of $G \times C^{p^{n-i+1}}$ onto $\left(G \times C^{p^{n-i}}\right) / C^{p^{n-1}}$ in which the element $g c^{\alpha p^{n-i+1}}$ of $G \times C^{p^{n-s+1}}$ maps onto the coset $g c^{\alpha p^{n-i}} C^{p^{n-1}}$ where $g$ is any element of $G$ and $c$ is some fixed generator of $C$. Clearly, the restriction of $\varphi_{i+1}$ to $G \times C^{p^{n-i+1}}$ is $\varphi_{i}$.

Let $\bar{\varphi}_{i}$ be the isomorphism of $\bar{G} \times \bar{C}_{1} \times \cdots \times \bar{C}_{i-1}$ onto $\left(\bar{G} \times \bar{C}_{1} \times \cdots \times \bar{C}_{i}\right) / \bar{C}_{1}$ in which the element $\bar{g} \bar{c}_{1}^{\alpha_{1}} \bar{c}_{2}^{\alpha_{2}} \cdots \bar{c}_{i-1}^{\alpha_{i-1}}$ maps into the coset $\bar{g} \bar{c}_{\mathbf{2}}^{\alpha_{1}} \bar{c}_{\mathbf{3}}^{\alpha_{2}} \cdots \bar{c}_{i}^{\alpha_{i-1}} \bar{C}_{1}$ where $\bar{c}_{1}, \cdots, \bar{c}_{n}$ are a fixed set of generators of $\bar{C}_{1}, \cdots, \bar{C}_{n}$, and $\bar{g}$ is any element of $\bar{G}$. Clearly, the restriction of $\bar{\varphi}_{i+1}$ to $\bar{G} \times \bar{C}_{1} \times \cdots \times \bar{C}_{i-1}$ is $\bar{\varphi}_{i}$.

By induction over $n$, we prove that there exist extensions $F_{1}, F_{2}, \cdots, F_{n}$ of $f=F_{0}$ to $G \times C^{p^{n-1}}, G \times C^{\boldsymbol{p}^{n-2}}, \cdots, G \times C$ respectively such that

(1) $F_{i}$ is an extension of $F_{i-1}$

(2) for all $X$ such that $G \times C^{p^{n-i}} \supseteq X \supseteqq C^{p^{n-1}}$,

$$
F_{i} X=\bar{\varphi}_{i} F_{i} \varphi_{i}^{-1} X \text {. }
$$

By Theorem 3.1, this holds for $n=1$ since condition (2) is trivially satisfied by any $F$ satisfying condition (1).

Suppose $F_{1}, \cdots, F_{n-1}$ exist satisfying these conditions. Define 
$F_{n} X=F_{n-1} X$ for all $X \subseteq G \times C^{p}$ and $F_{n} X=\bar{\varphi}_{n} F_{n-1} \varphi_{n}^{-1} X$ for all $X \supseteqq C^{n-1}$. This is consistent since for $G \times C^{p} \supseteqq X \supseteqq C^{p^{n-1}}$,

$$
\begin{aligned}
F_{n-1} X & =\bar{\varphi}_{n-1} F_{n-1} \varphi_{n-1}^{-1} X \quad \text { by (2) } \\
& =\bar{\varphi}_{n} F_{n-1} \varphi_{n}^{-1} X .
\end{aligned}
$$

By Lemma 5.5, $F_{n} X$ can be defined for $G \times C^{p} \neq X \neq C^{p^{n-1}}$ such that $F_{n}$ is an embedding of $G \times C . F_{n}$ clearly satisfies condition (1). By the definition of $F_{n}$ for $X \supseteq C^{p^{n-1}}$

$$
\begin{aligned}
F_{n} X & =\bar{\varphi}_{n} F_{n-1} \varphi_{n}^{-1} X \\
& =\bar{\varphi}_{n} F_{n} \varphi_{n}^{-1} X
\end{aligned}
$$

and condition (2) is satisfied. This completes the proof of the theorem.

\section{Abelian Groups}

If $G$ is an abelian $p$-group, then $G$ has cyclic subgroups $C_{1}, \cdots, C_{n}$ such that $G=C_{1} \times C_{2} \times \cdots \times C_{n}$. Let $\left|C_{i}\right|=p^{\lambda_{i}}$. Then the $\lambda_{i}$ are uniquely determined (up to order) by $G$. We may assume $\lambda_{1} \geqq \lambda_{2} \geqq \cdots \geqq \lambda_{n}$. We say that $G$ is a group of type $\left(\lambda_{1}, \lambda_{2}, \cdots, \lambda_{n}\right)$.

Lemma 6.1. Let $G$ be an abelian p-group of type $\left(\lambda_{1}, \lambda_{2}, \cdots, \lambda_{n}\right)$ with $\lambda_{3} \leqq 1$. Then $G$ has an L-embedding in the elementary abelian group.

PROOF. $G$ is the direct product of a group of type $\left(\lambda_{1}, \lambda_{2}\right)$ and of cycles of order $p$. By Corollary 4.4 , the group of type $\left(\lambda_{1}, \lambda_{2}\right)$ has a 1-canonical $L$ embedding, which, by Theorems 3.1 and 3.3 , can be extended to an $L$ embedding of $G$.

LemMa 6.2. Let $G$ be an abelian p-group of type $\left(\lambda_{1}, \lambda_{2}, \cdots, \lambda_{n}\right)$ with $\lambda_{2} \leqq 2$. Then $G$ has an L-embedding in the elementary abelian group.

Proof. By Theorem 5.6, it is sufficient to prove the existence for the case $\lambda_{1}=\lambda_{2}=\cdots=\lambda_{n}=2$.

There exists an integer $\alpha\left(\bmod p^{2}\right)$ such that $a \rightarrow a^{\alpha}$ is an automorphism of order $p-1$ of the cyclic group $\{a\}$ of order $p^{2}$. Let $\theta$ be an integer $(\bmod p)$ with $\alpha \equiv \theta(\bmod p)$. We define the symbol ${ }^{*}$ by $\alpha^{*}=0, \theta^{*}=0$. Then for any $x$, there exist $r, s$ with $a^{x}=a^{\alpha^{r+s p}}$ where $r={ }^{*}, 0,1, \cdots, p-2$ and $s=0,1, \cdots, p-1$.

We construct an $L$-embedding of $G=\left\{a_{1}, \cdots, a_{n}\right\}$ of type $(2,2, \cdots, 2)$ in the elementary abelian group (written additively) with basis $x_{1}, y_{1}, x_{2}, y_{2}, \cdots, x_{n}, y_{n}$. Every element $g \in G$ can be written in the form

$$
g=\prod_{i=1}^{n} a_{i}^{\alpha^{r_{i}}+s_{i} D}
$$

Put $\quad f\{g\}=\left\{\sum_{i=1}^{n} \theta^{r_{i}} x_{i}, \sum_{i=1}^{n}\left(s_{i} x_{i}+\theta^{r_{i}} y_{i}\right)\right\} \quad$ for $g=\prod_{i=1}^{n} a_{i}^{\alpha^{r_{i}}+s_{i} D}$

$$
f\left\{g_{1}, \cdots, g_{k}\right\}=f\left\{g_{1}\right\} \cup \cdots \cup f\left\{g_{k}\right\} \text {. }
$$


We have to prove that $f$ so defined is one-to-one and that

$$
f A \supset f B \text { if and only if } A \supset B \text {. }
$$

(a) $f\{g\} \subseteq f\left\{g_{1}, \cdots, g_{k}\right\}$ if and only if $g \in\left\{g_{1}, \cdots, g_{k}\right\}$.

Proof. Put

$$
g_{i}=\prod_{j=1}^{n} a_{j}^{\alpha^{\tau_{i j}}+s_{i j} \nu}, g=\prod_{j=1}^{n} a_{j}^{\alpha^{r_{j}+s_{j} \nu} .}
$$

$g \in\left\{g_{1}, \cdots, g_{k}\right\}$ if and only if there exist $u_{i}, v_{i}$ with

$$
g=\prod_{i=1}^{k} g_{1}^{\alpha^{u_{i}}+v_{i} p}
$$

that is, if and only if there exist $u_{i}, v_{i}$ with

$$
\begin{aligned}
\alpha^{r_{j}}+s_{j} p & =\sum_{i=1}^{k}\left(\alpha^{r_{i j}}+s_{i j} p\right)\left(\alpha^{u_{i}}+v_{i} p\right) \\
& =\sum_{i=1}^{k}\left(\alpha^{u_{i}} \alpha^{r_{i j}}+p\left(\alpha^{u_{i}} s_{i j}+v_{i} \alpha^{\tau_{i j}}\right)\right)\left(\bmod p^{2}\right) j=1,2, \cdots, n .
\end{aligned}
$$

This is equivalent to

$$
\begin{aligned}
& \theta^{r_{j}}=\sum_{i=1}^{k} \theta^{u_{i}} \theta^{r_{i s}} \quad(\bmod p) \quad j=1,2, \cdots, n \\
& s_{j}=\sum_{i=1}^{k}\left(\theta^{u_{i}} s_{i j}+v_{i} \theta^{r_{i j}}\right)(\bmod p) \quad j=1,2, \cdots, n .
\end{aligned}
$$

This holds if and only if

$$
\begin{aligned}
& \sum_{j=1}^{n} \theta^{r_{j}} x_{j}=\sum_{i=1}^{k} \theta^{u_{i}} \sum_{j=1}^{n} \theta^{r_{i j}} x_{j} \\
& \sum_{j=1}^{n}\left(s_{j} x_{j}+\theta^{r_{j}} y_{j}\right)=\sum_{i=1}^{k} \theta^{u_{i}} \sum_{j=1}^{n}\left(s_{i j} x_{j}+\theta^{r_{i j}} y_{j}\right)+\sum_{i=1}^{k} v_{i} \sum_{j=1}^{n} \theta^{r_{i j}} x_{j} .
\end{aligned}
$$

This is the condition that $f\{g\} \subseteq f\left\{g_{1}, \cdots, g_{k}\right\}$.

(b) From (a), it follows that $f\{g\}$ is independent of the choice of the generator $g$ of $\{g\}$.

(c) From (a), it follows that for all $A \subseteq G$,

$$
f A=\cup\{f X \mid X \text { cyclic, } X \subseteq A\} .
$$

Therefore $f$ is one-to-one and $f A \supset f B$ if and only if $A \supset B$.

THEOREM 6.3. Let $G$ be an abelian p-group of type $\left(\lambda_{1}, \lambda_{2}, \cdots, \lambda_{n}\right)$ with $\lambda_{1}=\lambda_{2}=\lambda_{3} \doteq 2$. Then $G$ has a unique L-embedding in the elementary abelian group.

Proof. By Lemma 6.2, an $L$-embedding exists. Let $f_{1}$ and $f_{2}$ be $L$-embeddings of $G$ in the elementary abelian group $H$. We have to prove that $f_{1}$ 
and $f_{2}$ are equivalent. By Theorem 3.2, it is sufficient to consider the case $\lambda_{1}=\lambda_{2}=\cdots=\lambda_{n}=2$.

Let $G=\left\{a_{1}, \cdots, a_{n}\right\}$. Since $n \geqq 3$, a basis $x_{1}, \cdots, x_{n}, y_{1}, \cdots, y_{n}$ of $H$ can be chosen such that

$$
\begin{gathered}
\left\{x_{i}\right\}=f_{1}\left\{a_{i}^{\text {D }}\right\} \\
\left\{x_{i}, y_{i}\right\}=f_{1}\left\{a_{i}\right\}
\end{gathered}
$$

and $a_{i}^{p} \rightarrow x_{i}$ is an isomorphism of $\left\{a_{1}^{p}, \cdots, a_{n}^{p}\right\}$ onto $\left\{x_{1}, \cdots, x_{n}\right\}$ inducing $f_{1}$ on $\left\{a_{1}^{p}, \cdots, a_{n}^{\text {p }}\right\}, a_{i}\left\{a_{1}^{p}, \cdots, a_{n}^{\text {p }}\right\} \rightarrow y_{i}+\left\{x_{1}, \cdots, x_{n}\right\}$ is an isomorphism of $G / \Phi(G)$ onto $H / f_{1} \Phi(G)$ inducing $f_{1}$ on $G / \Phi(G)$. Then $f_{1}\left\{a_{1} a_{2} \cdots a_{n}\right\}=$ $\left\{\left(x_{1}+x_{2}+\cdots+x_{n}\right), \quad\left(y_{1}+y_{2}+\cdots+y_{n}+\alpha_{1} x_{1}+\cdots+\alpha_{n} x_{n}\right)\right\}$ for some $\alpha_{1}, \cdots, \alpha_{n}$. We can choose $y_{1}, \cdots, y_{n}$ such that $\alpha_{1}=\alpha_{2}=\cdots=\alpha_{n}=0$ since we can replace $y_{i}$ by $y_{i}+\alpha_{i} x_{i}$.

Similarly, we can choose a basis $x_{1}^{\prime}, \cdots, x_{n}^{\prime}, y_{1}^{\prime}, \cdots, y_{n}^{\prime}$ such that

$$
\begin{aligned}
& f_{2}\left\{a_{i}^{p}\right\}=\left\{x_{i}^{\prime}\right\} \\
& f_{2}\left\{a_{i}\right\}=\left\{x_{i}^{\prime}, y_{i}^{\prime}\right\} \\
& f_{2}\left\{a_{1} a_{2} \cdots a_{n}\right\}=\left\{\left(x_{1}^{\prime}+x_{2}^{\prime}+\cdots+x_{n}^{\prime}\right),\left(y_{1}^{\prime}+y_{2}^{\prime}+\cdots+y_{n}^{\prime}\right)\right\}
\end{aligned}
$$

and $a_{i}^{p} \rightarrow x_{i}^{\prime}$ is an isomorphism inducing $f_{2}$ on $\left\{a_{1}^{p}, \cdots, a_{n}^{p}\right\}$, $a_{i}\left\{a_{1}^{p}, \cdots, a_{n}^{p}\right\} \rightarrow y_{i}^{\prime}+\left\{x_{1}^{\prime}, \cdots, x_{n}^{\prime}\right\}$ is an isomorphism inducing $f_{2}$ on $G / \Phi(G)$. Let $\beta$ be the automorphism of $H: \beta x_{i}^{\prime}=x_{i}, \beta y_{i}^{\prime}=y_{i}$. Then $\beta f_{2} X=f_{1} X$ for all $X \supseteqq \Phi(G)$ and for $X=\left\{a_{i}\right\},\left\{a_{1} a_{2} \cdots a_{n}\right\}$. Let $Z \subset G$ be any subgroup such that $Z \neq \Phi(G), Z \neq \Phi(G)$. Then

$$
\Phi(Z \cup \Phi(G))=(Z \cup \Phi(G))^{p}=Z^{p} \cup(\Phi(G))^{p}=Z^{p} \subset G^{p}=\Phi(G) .
$$

Therefore there exist $X, Y$ such that $X \supset \Phi(G) \supset Y, X \supset Z \supset Y$ and $X / Y$ is elementary abelian. Thus $\beta f_{2}$ maps $L(X / Y)$ onto $f_{1} L(X / Y)$ and there exists $Z^{\prime}$ such that $\beta f_{2} Z=f_{1} Z^{\prime}$. Therefore for all $A \subseteq G$, there exists $B \subseteq G$ such that $\beta f_{2} A=f_{1} B$.

Since $\beta f_{2} L(G)=f_{1} L(G)$, we can form the mapping $f_{1}^{-1} \beta f_{2}$ of $L(G)$ onto itself. This is a lattice automorphism of $G$. But by a theorem of Baer ([1] page 35 Theorem 2), there exists an automorphism $\alpha$ of $G$ inducing $f_{1}^{-1} \beta f_{2}$. Therefore $\beta f_{2}=f_{1} \alpha$. But from the construction of $\beta$,

$$
\begin{aligned}
\alpha\left\{a_{i}\right\} & =\left\{a_{i}\right\} \\
\alpha\left\{a_{1} a_{2} \cdots a_{n}\right\} & =\left\{a_{1} a_{2} \cdots a_{n}\right\} .
\end{aligned}
$$

Hence $\alpha$ has the form $\alpha g=g^{r}$ for all $g \in G$, where $r$ is independent of $g$. Therefore $\alpha$ induces the identity lattice automorphism and therefore $\beta f_{2}=f_{1}$.

LEMMA 6.4. There exists no L-embedding of an abelian group of type $(3,3, \dot{2})$ in the elementary abelian group.

Proof. Let $G=\{a, b\}, a^{p^{2}}=b^{v^{2}}=1$ be an abelian group of type $(3,2)$. Let $f$ be any $L$-embedding of $G$ in the elementary abelian group. It is suffi- 
cient to prove that $f$ is not 1-2-, 3-canonical. Suppose $f$ is 1- and 2-canonical. Take a basis $e_{1}, e_{2}, e_{3}$ of $f\{a\}$ such that

$$
f\left\{a^{p^{2}}\right\}=\left\{e_{1}\right\}, \quad f\left\{a^{p}\right\}=\left\{e_{1}, e_{2}\right\} .
$$

Consider the chain of projectivities

$$
c:\{a\} \rightarrow\{a, b\} /\{b\} \rightarrow\{a b\} \rightarrow\{a, b\} /\left\{a^{p} b\right\} \rightarrow\{a\} .
$$

In this, $a \rightarrow a\{b\} \rightarrow a b \rightarrow a b\left\{a^{p} b\right\} \rightarrow a^{1-p}$. If $A$ is the matrix of $\bar{\alpha}(\bar{c})$ then from the first canonicity condition, $A$ is of the form

$$
A=\left[\begin{array}{lll}
1 & x & y \\
0 & 1 & z \\
0 & 0 & 1
\end{array}\right]
$$

(a) Case $p>2$. The automorphism $\alpha(c)$ is of order $p^{2}$.

$$
\begin{array}{rlrl}
A^{p}-I & =(A-I)^{p} & & \text { since the matrix is over the field of } p \\
& =0 & & \text { elements } \\
& \text { since } p \geqq 3 \text { and }(A-I)^{3}=0 .
\end{array}
$$

Put $\gamma=c^{p}$. Then $\alpha(\gamma) \neq 1$ but $\bar{\alpha}(\bar{\gamma})=1$ and $f$ is not 3-canonical.

(b) Case $p=2$. $\alpha(c) a=a^{-1}$.

Consider the restrictions $c^{\prime}, c^{\prime \prime}$ of $c$ to chains of projectivities on factors of order 4, starting at $\left\{a^{2}\right\}$ and $\{a\} /\left\{a^{4}\right\}$. Let $A^{\prime}, A^{\prime \prime}$ be the matrices of $\bar{\alpha}\left(\bar{c}^{\prime}\right)$, $\bar{\alpha}\left(\bar{c}^{\prime \prime}\right)$ respectively. Then

$$
A^{\prime}=\left[\begin{array}{ll}
1 & x \\
0 & 1
\end{array}\right] \text { and } A^{\prime \prime}=\left[\begin{array}{ll}
1 & z \\
0 & 1
\end{array}\right] \text {. }
$$

$\alpha\left(c^{\prime}\right) a^{2}=\left(a^{2}\right)^{-1}$ and $\alpha\left(c^{\prime}\right) \neq 1$. Therefore $x \neq 0$ since $f$ is 2-canonical. $\alpha\left(c^{\prime \prime}\right) a\left\{a^{4}\right\}=a^{-1}\left\{a^{4}\right\}$ and $\alpha\left(c^{\prime \prime}\right) \neq 1$. Therefore $z \neq 0$. Therefore $A^{2} \neq I$. Put $\gamma=c^{2}$. Then $\alpha(\gamma)=1$ but $\bar{\alpha}(\bar{\gamma})$ has matrix $A^{2} \neq I$ and $f$ is not 3-canonical.

Theorem 6.5. There exists an L-embedding of the abelian p-group $G$ in the elementary abelian group if and only if $G$ has no subgroup of type $(3,3,2)$.

Proof. If an $L$-embedding of $G$ in the elementary abelian group exists, then $G$ has no subgroup of type $(3,3,2)$ by Lemma 6.4 .

Suppose $G$ has no subgroup of type $(3,3,2)$. Let $G$ be of type $\left(\lambda_{1}, \cdots, \lambda_{r}\right)$. Either $\lambda_{3} \leqq 1$ and $G$ has an $L$-embedding in the elementary abelian group by Lemma 6.1, or $\lambda_{2} \leqq 2$ and by Lemma $6.2 G$ has an $L$-embedding in the elementary abelian group.

For abelian.p-groups with a subgroup of type $(3,3,2)$, we have the stronger result:

THEOREM 6.6. Let $G$ and $H$ be abelian groups of the same prime power order. Suppose $G$ has a subgroup of type $(3,3,2)$ and that $f$ is an $L$-embedding of $G$ in $H$. Then $G \cong H$. 
Proof. The result holds for $G$ of type $(3,3,2)$ since it cannot have an $L$-embedding in a group which is $L$-embeddable in the elementary abelian group.

Take a direct decomposition $G=X_{1} \times X_{2} \times \cdots \times X_{n}$ of $G$ with $X_{i}$ cyclic. It is sufficient to prove that $f X_{i}$ is cyclic.

If $X_{i}$ is of order $p$, then trivially $f X_{i}$ is cyclic. If $X_{i}$ is of order $p^{2}$ or $p^{3}$, then there exist subgroups $A \subseteq X_{j}, B \subseteq X_{k}(i, j, k$ distinct) such that $X_{i} \times A \times B$ is of type $(3,3,2)$ and therefore $f X_{i}$ is cyclic.

Suppose $X_{i}$ is of order $p^{r}, r>3$. We use induction over $r$. There exist $A \subseteq X_{j}, B \subseteq X_{k}\left(i, j, k\right.$ distinct) such that $X_{i} \times A \times B$ is of type $(r, 3,2)$. We assume that the theorem holds for groups of type $(r-1,3,2), r>3$. $X_{i}^{p} \times A \times B$ and $\left(X_{i} \times A \times B\right) / X_{i}^{p^{r-1}}$ are of type $(r-1,3,2)$ and therefore $f\left(X_{i}^{p}\right)$ and $f X_{i} / f\left(X_{i}^{p^{r-1}}\right)$ are cyclic. But

$$
\Phi\left(f X_{i}\right) \supseteqq \Phi\left(f\left(X_{i}^{p}\right)\right) \supseteqq f\left(X_{i}^{p^{r-1}}\right) .
$$

Therefore $f X_{i} / \Phi\left(f X_{i}\right)$ is cyclic. Hence $f X_{i}$ is cyclic.

\section{Non-Abelian Groups}

Clearly, if a $p$-group $G$ is lattice isomorphic to an abelian $p$-group $A$, then $G$ has an $L$-embedding in the elementary abelian group if and only if $A$ has. We use this to extend the result of Theorem 6.5 to $p$-groups with modular lattices of subgroups.

Iwasawa $([1], p, 15)$ in determining the structure of non-Hamiltonian modular $p$-groups, shows that such a group $G$ has a basis $a_{1}, \cdots, a_{k}$. If $a_{i}$ has order $p^{\lambda_{i}}, \lambda_{1} \geqq \lambda_{2} \geqq \cdots \geqq \lambda_{k}$, we say $G$ is of type $\left(\lambda_{1}, \cdots, \lambda_{k}\right)$. By a theorem of Jones ([2], p. 554, Theorem 3.13), $G$ is lattice isomorphic to an abelian $p$-group of the same type.

THeOREM 7.1. (a) Let $G$ be a non-Hamiltonian modular p-group. Then $G$ has an L-embedding in the elementary abelian group of the same order if and only if $G$ contains no subgroup of type $(3,3,2)$.

(b) Let $G$ be a Hamiltonian p-group. Then $G$ has an L-embedding in the elementary abelian group of the same order.

Proof. (a) has already been proved. Suppose $G$ is a Hamiltonian $p$-group. Then $G$ is the direct product of a quaternion group of order 8 with an elementary abelian 2-group. By Theorems 4.2, 3.1, $G$ has an $L$-embedding in the elementary abelian group of the same order.

Lazard ([3], p. 176, Theorem 4.6) has given a construction based on the Baker-Hausdorff formula which defines, for any p-group $G$ such that all subgroups generated by three (not necessarily distinct) elements have class strictly less than $p$, an addition and Lie multiplication on the elements of $G$ making $G$ a Lie-ring $\mathscr{L}(G)$ with respect to this addition and Lie multiplication. In this construction, subgroups correspond to subrings, the element 
$1 \epsilon G$ corresponds to $0 € \mathscr{L}(G)$ and $x^{p^{*}}=1$ in $G$ if and only if $p^{*} x=0$ in $\mathscr{L}(G)$. We denote by $A(G)$ the additive group of $\mathscr{L}(G)$. Since $G$ is regular, we can define the type invariants as done by $\mathrm{P}$. Hall ([4], p. 79-81). When $G$ is modular, these invariants coincide with those used above. Let $\Omega_{\alpha}(G)=\left\{x \mid x \in G, x^{p^{\alpha}}=1\right\}$. Then $A\left(\Omega_{\alpha}(G)\right)=\Omega_{\alpha}(A(G))$. Hence $G$ and $A(G)$ have the same invariants. If $X \subseteq G$, then $A(X) \subseteq A(G)$. Thus the mapping $f$ defined by $f X=A(X)$ is an $L$-embedding of $G$ in $A(G)$.* $^{*}$ Hence we have

THEOREM 7.2. Suppose $G$ is a p-group of class less than $p$. Then $G$ has an $L$-embedding in the abelian group with the same invariants.

THEOREM 7.3. Suppose $G$ is a p-group of class less than $p$ and with invariants $\left(\lambda_{1}, \cdots, \lambda_{k}\right), \lambda_{1} \geqq \lambda_{2} \geqq \cdots \geqq \lambda_{k}$ satisfying either $\lambda_{2} \leqq 2$ or $\lambda_{3} \leqq 1$. Then $G$ has an L-embedding in the elementary abelian group of the same order.

Using these results, we can generalize Theorem 6.6.

Theorem 7.4. Let $G$ be a modular $p$-group of order $p^{n}, p \geqq n$, and suppose that $G$ has a subgroup of type $(3,3,2)$. Suppose $G$ has an L-embedding in the group $H$ of the same order. Then $L(G) \cong L(H)$.

Proof. $G, H$ have class at most $n-1<p$. Thus $A(G), A(H)$ exist and $G$ has an $L$-embedding in $A(H)$. But $L(G) \cong L(A(G))$. Therefore $A(G)$ has an $L$-embedding in $A(H)$. By Theorem 6.6, $A(G) \cong A(H)$. Therefore $A(H)$ has an $L$-embedding in $H$. Therefore $L(H) \cong L(A(H)) \cong L(G)$.

I should like to express my thanks to Dr. G. Higman and Dr. G. E. Wall for guiding my research, and to the C.S.I.R.O. and the University of Sydney for financial assistance.

\section{References}

[1] Suzuki, M., Structure of a group and the structure of its lattice of subgroups. (Springer, 1956).

[2] Jones, A. W., The lattice isomorphisms of certain finite groups, Duke Math. J. 12 (1945), $541-560$.

[3] Lazard, M., Sur les groupes nilpotents et les anneaux de Lie, Ann. Sc. de l'Ecole Normale Superieure (1954), 101-190.

[4] Hall, P., A contribution to the theory of groups of prime-power order, Proc. London Math. Soc. (2) 36 (1933), 29-95.

[5] Wall, G. E., Some applications of the Eulerian functions of a finite group (submitted to this Journal).

Universität Tübingen

Germany

* For this result I am indebted to Dr. G. Higman. 